\title{
Interaction of Phosphoglycerate Kinase from Escherichia coli with Cibacron Blue
}

\author{
Keiichi KawaI, * Shinro TaKahashi and Yoshitomo EGUCHI \\ Department of Agricultural Chemistry, Faculty of Agriculture, \\ Hokkaido University, Kita-ku, Sapporo 060, Japan \\ Received October 29, 1980
}

\begin{abstract}
Phosphoglycerate kinase (ATP: 3-phospho-D-glycerate 1-phosphotransferase, EC 2.7.2.3) from Escherichia coli AHU 1520 was purified until electrophoretically homogeneous by a single procedure, using a linear gradient elution of 2,3-bisphosphoglycerate between 0 and $0.5 \mathrm{~mm}$ on a blue dextran-Sepharose 4B column chromatography. The phosphoglycerate kinase was inhibited by blue dextran competitively with respect to 3-phosphoglycerate, and noncompetitively with respect to ATP. The inhibition of the kinase by Cibacron Blue was competitive with respect to 3phosphoglycerate at both saturated and unsaturated levels of ATP; the inhibition by the dye toward ATP was shifted from a mixed-type at a saturated level of 3-phosphoglycerate to competitive-type at an unsaturated level. Further inhibition studies implied that two molecules of the dye were bound per molecule of phosphoglycerate kinase with an unsaturated level of ATP used as a fixed substrate. The shape of the difference spectrum of Cibacron Blue in the presence of the kinase was very similar to that of a difference spectrum generated upon addition of $\mathrm{KCl}$, but was markedly different from the shape of the spectrum produced by the addition of glycerol.
\end{abstract}

Inhibition studies of rabbit muscle and beef heart lactate dehydrogenases and yeast phosphoglycerate kinase by blue dextran or Cibacron Blue have shown that these dyes bind at the nucleotide binding site(s) on the enzymes. ${ }^{1 \sim 3)}$ Recently Beissner and Rudolph ${ }^{4)}$ have shown that the 1-amino-4-(4'-aminophenylamino)-anthraquinone 2,3-disulfonic acid portion of Cibacron Blue is a strong competitive inhibitor of hexokinase, lactate dehydrogenase, malate dehydrogenase and glucose 6-phosphate dehydrogenase with respect to the nucleotide substrates and interacts specifically with the nucleotide binding sites of these enzymes. Böhme et al. ${ }^{5)}$ and Stellwagen ${ }^{3)}$ have both proposed that this portion of the dye molecule is an adenine nucleotide analog. It appears that the portion is essential in the binding of Cibacron Blue to dehydrogenases or kinases.

* Present address: Department of Agricultural Chemistry, Faculty of Agriculture, Gifu University, Kakamigahara, Gifu 504, Japan.
Yeast phosphoglycerate kinase was shown to be inhibited by either blue dextran ${ }^{1)}$ or Cibacron Blue ${ }^{6)}$ competitively with respect to ATP, and it was suggested that it would bind with blue dextran-Sepharose at the ATP binding site. ${ }^{2,6)}$ Recently we have found that phosphoglycerate kinase of Lactobacillus plantarum was inhibited by Cibacron Blue noncompetitively with respect to ATP, but competitively with respect to 3-phosphoglycerate. $^{7}$ It was suggested that the 3phosphoglycerate binding site of Lactobacillus kinase is responsible for the adsorption of the kinase to blue dextran-Sepharose. Thus, it is questionable whether the ATP binding site on phosphoglycerate kinase is responsible for the adsorption of the kinase to blue dextranSepharose. Such differences underlie the need for caution in extending conclusions based on one enzyme to those from different sources. Therefore, it is of interest to study the adsorption mechanism of phosphoglycerate kinase from other sources to blue dextran-Sepharose.

In this study, we describe the interactions of 
phosphoglycerate kinase from $E$. coli with blue dextran and Cibacron Blue.

\section{MATERIALS AND METHODS}

Microorganism. E. coli AHU 1520 was used throughout this study. The cells were cultivated in $100 \mathrm{ml}$ of liquid medium in $500-\mathrm{ml}$ Sakaguchi flask at $37^{\circ} \mathrm{C}$ for $6 \mathrm{hr}$ on a reciprocal shaker. The composition of the medium used was as follows: $1 \%$ Polypeptone, $1 \%$ meat extract, $0.5 \%$ glucose and $0.5 \% \mathrm{NaCl}$. The cells were harvested by centrifugation and washed with cold deionized water. The washed cells were suspended in five volumes of $10 \mathrm{~mm}$ Tris- $\mathrm{HCl}$ buffer $(\mathrm{pH} 7.5)$ and ruptured by sonic oscillation for $10 \mathrm{~min}$ at $0^{\circ} \mathrm{C}$. The cellular debris was removed by centrifugation for $10 \mathrm{~min}$ at $12,000 \times g$.

Enzyme assay. The activity of phosphoglycerate kinase was determined spectrophotometrically. ${ }^{7)}$ One unit of enzyme was defined as the amount of enzyme forming $1 \mu \mathrm{mol}$ of 1,3-bisphosphoglycerate per min.

Protein assay. Protein content was determined by the method of Lowry $e t a l^{8)}$ using bovine serum albumin as a standard.

Polyacrylamide gel disc electrophoresis. Disc electrophoresis was carried out with 7\% polyacrylamide gel in Trisglycine buffer at $\mathrm{pH}$ 9.4. Electrophoresis was performed at room temperature, applying a constant current of $3 \mathrm{~mA}$ per tube. After the run, the gels were stained with $1 \%$ Amido Black $10 \mathrm{~B}$ in $7 \%$ acetic acid. For the determination of enzyme activity in a gel, the gel was cut into $2-\mathrm{mm}$ pieces and each piece was placed in $0.5 \mathrm{ml}$ of $10 \mathrm{~mm}$ Tris$\mathrm{HCl}$ buffer. After remaining overnight at $4^{\circ} \mathrm{C}$, the enzyme activity in the buffer was determined under standard assay conditions.

Chemicals. Glyceraldehyde 3-phosphate dehydrogenase from rabbit muscle, 3-phosphoglycerate and 2,3-bisphosphoglycerate were from Boehringer Mannheim GmbH., West Germany. Blue dextran-Sepharose 4B was prepared by the method of Ryan and Vestling. ${ }^{9)}$ ATP, ADP, AMP, GDP, GMP, NADP and NADPH were purchased from Kyowa Hakko Kogyo Co., Ltd., Tokyo. NAD and NADH were the products of Oriental Yeast Co., Ltd., Tokyo. GTP, ITP and IDP were obtained from Sigma Chemicals Co., St. Louis. IMP was a generous gift from Takeda Chemical Industries, Ltd., Osaka. Cibacron Blue F3GA was from Polysciences, Inc.

\section{RESULTS}

Elution of phosphoglycerate kinase from blue dextran-Sepharose with various ligands

The phosphoglycerate kinase of $E$. coli AHU 1520 was found to bind to blue dextranSepharose. The adsorbed enzyme was quantitatively eluted with ether $1 \mathrm{~mm}$ ATP or $1 \mathrm{~mm} 3-$ phosphoglycerate. When the kinase was eluted with a linear gradient of either $\mathrm{KCl}$ or 3phosphoglycerate, the concentrations of $\mathrm{KCl}$ and 3-phosphoglycerate in the peak fraction of enzyme activity were approximately $0.3 \mathrm{M}$ and $0.5 \mathrm{~mm}$, respectively. In addition, the kinase

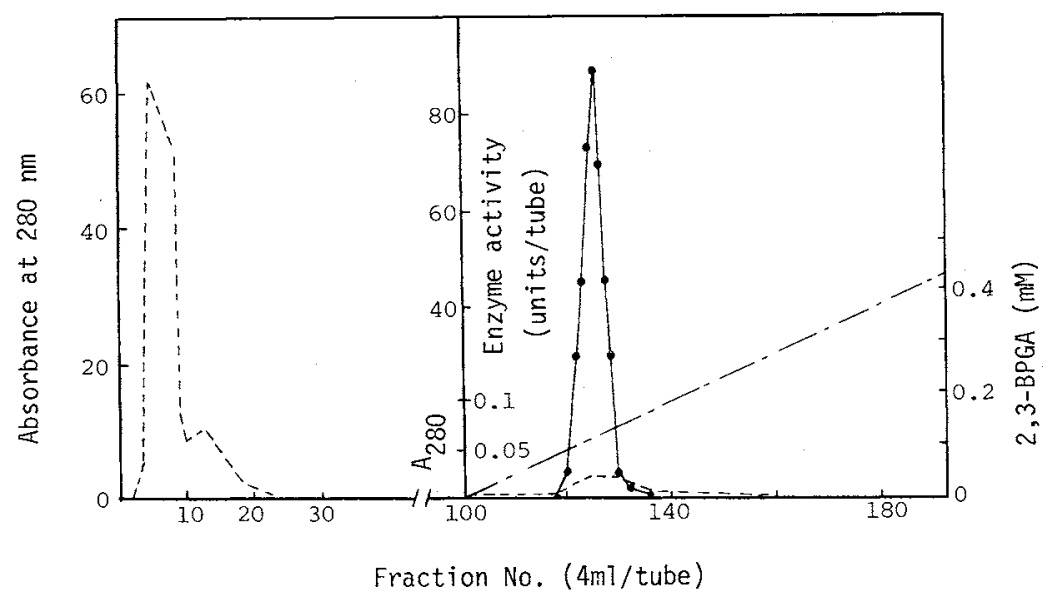

FIG. 1. Elution Profile of Phosphoglycerate Kinase with a Linear Gradient of 2,3-Bisphosphoglycerate from Blue Dextran-Sepharose.

The crude extract $(6 \mathrm{ml})$ was applied to a column $(2 \times 24 \mathrm{~cm})$ of blue dextran-Sepharose. Flow rate was $22.4 \mathrm{ml}$ per hr. - phosphoglycerate kinase activity; ---, absorbance at $280 \mathrm{~nm}$. 
Table I. Purification of E. coli Phosphoglycerate Kinase

\begin{tabular}{lccccc}
\hline \multicolumn{1}{c}{ Fraction } & $\begin{array}{c}\text { Total } \\
\text { activity } \\
\text { (units) }\end{array}$ & $\begin{array}{c}\text { Total } \\
\text { protein } \\
\text { (mg) }\end{array}$ & $\begin{array}{c}\text { Specific } \\
\text { activity } \\
\text { (units/mg protein) }\end{array}$ & $\begin{array}{c}\text { Yield } \\
(\%)\end{array}$ & $\begin{array}{c}\text { Purification } \\
\text { (fold) }\end{array}$ \\
\hline Crude extract & 492.7 & 210 & 2.35 & 100 & 1 \\
Blue dextran eluate & 310.9 & 0.236 & 1317.5 & 63.1 & 561 \\
\hline
\end{tabular}

can be eluted from the blue dextran column with 2,3-bisphosphoglycerate, ADP, GTP, GDP, ITP and IDP in good yields ( $>80 \%$ ). Although AMP and GMP did not elute the phosphoglycerate kinase obtained from $L$. plantarum AHU 1047, the kinase from E. coli AHU 1520 was eluted from the blue dextranSepharose column with these mononucleotides

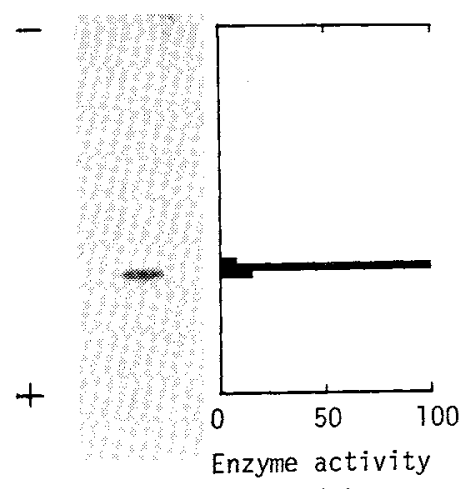

$(\%)$

FIG. 2. Polyacrylamide Gel Disc Electrophoresis of the Purified Phosphoglycerate Kinase.

The purified enzyme $(16.3 \mu \mathrm{g})$ was subjected to $7 \%$ polyacrylamide gel disc electrophoresis at $\mathrm{pH} 9.4$.

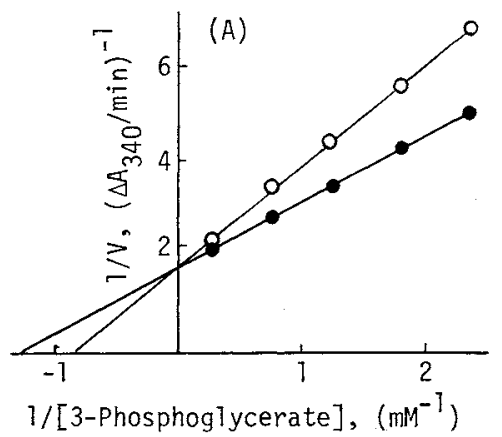

to some extent. Furthermore, NADP, NADPH and NADH eluted the kinase well $(>80 \%)$, but NAD was less effective $(26 \%)$. Considering these results, we attempted to purify the phosphoglycerate kinase of $E$. coli AHU 1520. The kinase was purified by a singlestep procedure using a linear gradient of 2,3bisphosphoglycerate between 0 and $0.5 \mathrm{~mm}$. The elution profile is shown in Fig. 1. The kinase was eluted as a sharp peak. Active fractions (fraction nos. 122-128) were pooled. As shown in Table I, the kinase was purified 561 -fold with a $63 \%$ yield. The purified enzyme migrated as a single electrophoretic protein band when subjected to $7 \%$ polyacrylamide gel electrophoresis at $\mathrm{pH} 9.4$, and the kinase activity comigrated with the protein band. Both of these results are illustrated in Fig. 2.

\section{Effects of blue dextran and Cibacron Blue on phosphoglycerate kinase}

The effect of blue dextran on the activity of $E$. coli phosphoglycerate kinase is shown in Fig. 3. The E. coli kinase was found to be inhibited by blue dextran noncompetitively

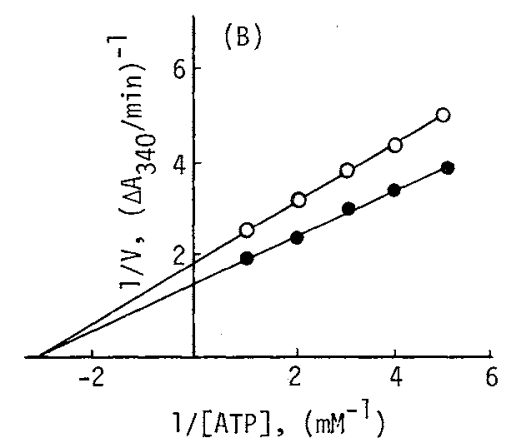

FrG. 3. Effect of Blue Dextran on the Activity of Phosphoglycerate Kinase.

The reactions were carried out in the absence $(O)$ and presence $(O)$ of blue dextran. The concentration of blue dextran was $98 \mu \mathrm{M}$ as a blue chromophore concentration. The concentrations of 3-phosphoglycerate (A) and ATP $(B)$ were varied as indicated. 

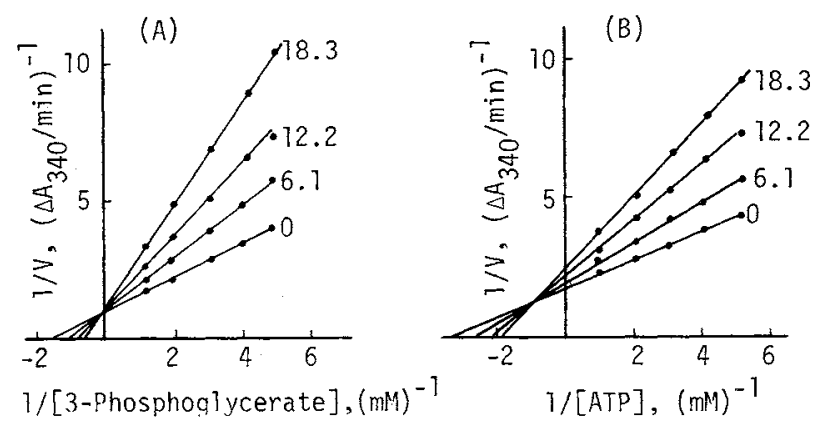

FIG. 4. Plots of the Reciprocal of Initial Velocity against the Reciprocal of the Concentration of 3Phosphoglycerate (A) or ATP (B) at a Saturated Level of Fixed Substrate in the Absence and Presence of Cibacron Blue.

The numbers in the figure represent Cibacron Blue concentration $(\mu \mathrm{M})$.

(A): The concentration of ATP was held constant at $2 \mathrm{~mm}$.

(B): The concentration of 3-phosphoglycerate was held constant at $3.3 \mathrm{~mm}$.

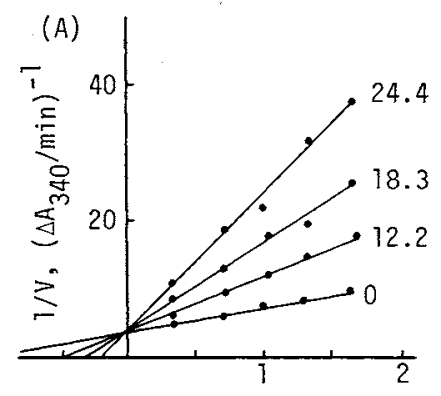

$1 /[3-$ Phosphoglycerate $],(\mathrm{mM})^{-1}$

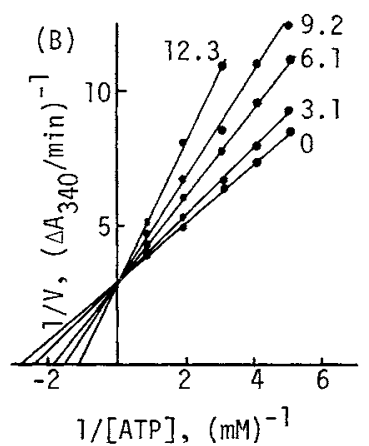

FIG. 5. Plots of the Reciprocal of Initial Velocity against the Reciprocal of the Concentration of 3Phosphoglycerate (A) or ATP (B) at an Unsaturated Level of Fixed Substrate in the Absence and Presence of Cibacron Blue.

The numbers in the figure represent Cibacron Blue concentration $(\mu \mathrm{M})$.

(A): The concentration of ATP was held constant at $0.2 \mathrm{~mm}$.

(B): The concentration of 3-phosphoglycerate was held constant at $0.6 \mathrm{~mm}$.

with respect to ATP, but competitively with respect to 3-phosphoglycerate. Next the effect of Cibacron Blue, a chromophore of blue dextran, on the activity of $E$. coli kinase was examined. The results obtained under a saturated level of each fixed substrate are shown in Fig. 4. Cibacron Blue showed competitive inhibition with respect to 3-phosphoglycerate and mixed-type inhibition with respect to ATP. Replotting the slope of the data in Fig. 4(A) gave a straight line (Fig. 6). ${ }^{10,11)}$ However, with an unsaturated level of a fixed substrate Cibacron Blue was a competitive inhibitor of both 3-phosphoglycerate and ATP, as shown in Fig. 5. When replotted, the

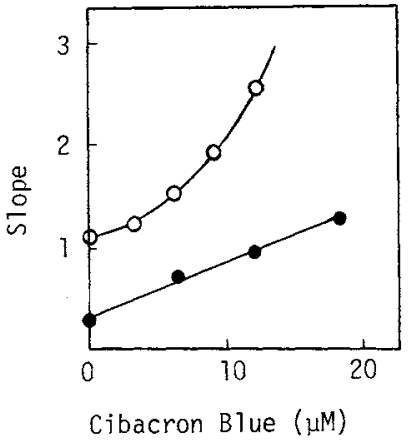

FIG. 6. Replots of the Slopes with Respect to Cibacron Blue Concentration.

The data (O) from Fig. 4(A) and those (O) from Fig. 5(A) were replotted. 


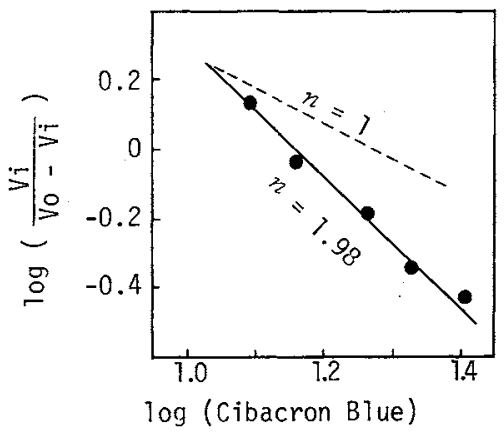

FIG. 7. Phosphoglycerate Kinase Activity as a Function of Cibacron Blue Concentration.

The reactions were carried out with an unsaturated level of ATP as a fixed substrate. The curve can be represented by Hill's empirical equation:

$$
\log \left(\frac{V i}{V o-V i}\right)=\log (K)-n \log (\text { Cibacron Blue) }
$$

$n$ : Hill's coefficient.

Vo: The initial velocity in the absence of Cibacron Blue.

$V i$ : The initial velocity in the presence of Cibacron Blue.

$K$ : Constant.

slope showed a nonlinear curve, which was found to fit a parabola (Fig. 6). ${ }^{10,11)}$ Furthermore, as shown in Fig. 7, the plot of enzyme activity as a function of the dye concentration gave a straight line, and the Hill's coefficient $(n)^{12,13)}$ was calculated to be 1.98 . These results indicate that there are at least two Cibacron Blue binding sites on $E$. coli phosphoglycerate kinase.

\section{Difference spectroscopy studies}

The difference spectrum of the titration of E. coli phosphoglycerate kinase with Cibacron Blue is shown in Fig. 8(A). It has a positive maximum at $680 \mathrm{~nm}$ and an isosbestic point at $646 \mathrm{~nm}$. This spectrum is similar to the difference spectrum of Cibacron Blue produced by titration with $\mathrm{KCl}$, but not to that produced with $50 \%$ glycerol (Fig. 8(B)). As shown in Fig. 9, the difference extinction at $680 \mathrm{~nm}$ increases in a hyperbolic manner with increasing the dye concentration. This indicates the formation of an enzyme-dye complex. Addition of either 3-phosphoglycerate or ATP

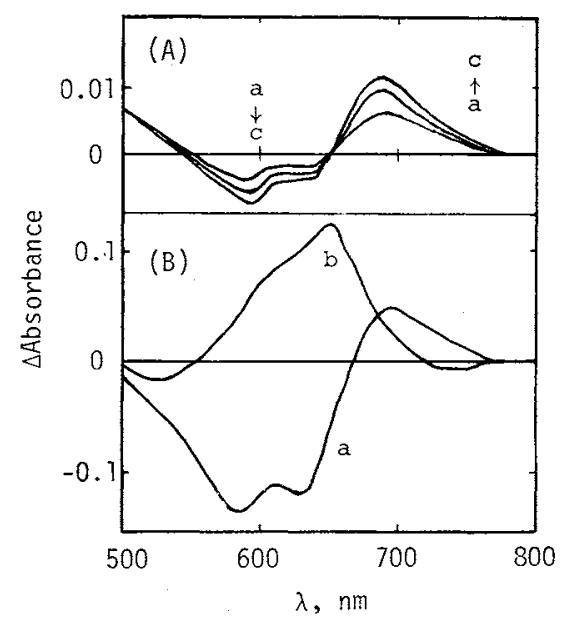

FIG. 8. Difference Spectra of Cibacron Blue in the Presence of Phosphoglycerate Kinase, $\mathrm{KCl}$ or Glycerol.

(A): Protein difference spectra. The sample cuvette contained enzyme $(7.0 \mu \mathrm{M})$, while both the sample and reference cuvettes contained (a) $9.2 \mu \mathrm{M}$, (b) $18.2 \mu \mathrm{M}$ and (c) $46 \mu \mathrm{m}$ dye. The solvent was $10 \mathrm{~mm}$ Tris-HCl buffer (pH 7.5).

(B): $\mathrm{KCl}$ and glycerol difference spectra. The sample cuvette contained (a) $45 \mathrm{~mm} \mathrm{KCl}$ or (b) $50 \%$ glycerol, while both the sample and reference cuvettes contained $171.6 \mu \mathrm{M}$ dye. The solvent was water.

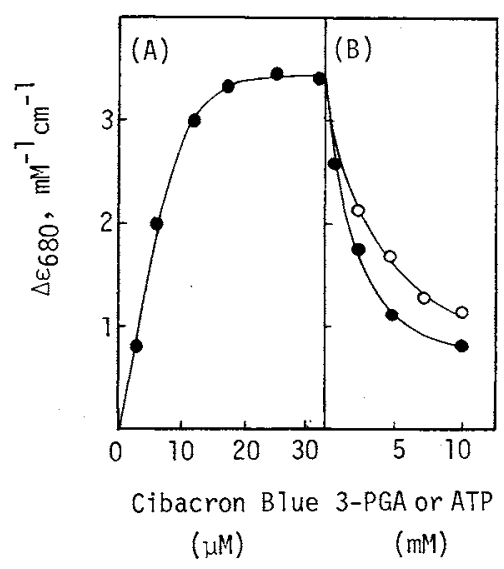

FIG. 9. Spectral Measurements of Enzyme-Dye Complexes.

(A): Difference spectral titration of phosphoglycerate kinase with Cibacron Blue. The initial enzyme concentration was $5.3 \mu \mathrm{M}$.

(B): Difference spectral titration of enzyme-dye complex with ATP (O) or 3-phosphoglycerate $(\bigcirc)$. The solvent was $10 \mathrm{~mm}$ Tris-HCl buffer (pH 7.5). Extinction values were corrected for dilution. 
to the complex results in a progressive decrease in the extinction, which is also shown in Fig. 9.

\section{DISCUSSION}

E. coli phosphoglycerate kinase was easily eluted by a low concentration of adenine nucleotide, guanine nucleotide or pyridine nucleotide. It should be noted that $E$. coli kinase was also eluted by 3-phosphoglycerate, as well as by ATP. In addition, the kinases of other strains of $E$. coli were also eluted with 3phosphoglycerate. Similar results were obtained for the phosphoglycerate kinase of several lactic acid bacteria ${ }^{14)}$ and of yeast. ${ }^{2,3,15)}$ Thus, the elution of phosphoglycerate kinase by a low concentration of 3-phosphoglycerate seems to be a general phenomenon. The elution of $E$. coli kinase required a concentration of $\mathrm{KCl}$ about three orders of magnitude greater than that of 3-phosphoglycerate, indicating that the interaction of the kinase with blue dextran-Sepharose is biospecific.

We obtained apparently electrophoretically homogeneous phosphoglycerate kinase from a crude extract of E. coli AHU 1520 in $63 \%$ yield by a single-step purification procedure using a linear gradient of 2,3-bisphosphoglycerate. The conventional purification procedure for this enzyme from $E$. coli involves five steps, and only $28 \%$ of the original amount of enzyme is recovered. ${ }^{16)}$ The purification procedure presented in this study may be very useful for the purification of phosphoglycerate kinases from other sources.

The spectral titrations revealed that the Cibacron Blue-phosphoglycerate kinase complex in solution was dissociated by the addition of either 3-phosphoglycerate or ATP. It is likely that the free dye specifically combined with the kinase at its active sites.

Thompson and Stellwagen ${ }^{2)}$ have shown that the difference spectrum produced by the addition of lactate dehydrogenase from rabbit muscle to Cibacron Blue solution is quite similar to the difference spectrum resulting from the presence of $50 \%$ ethylene glycol in the dye solution. This is, presumably, due to insertion of the dye into the hydrophobic pockets of the NAD binding sites. In the case of $E$. coli phosphoglycerate kinase, however, the difference spectrum of Cibacron Blue in the presence of the kinase was similar to that in the presence of $\mathrm{KCl}$, but not to that of $50 \%$ glycerol. Glycerol has a dielectric constant of 42.5 , indicating that it is an appropriate hydrophobic solvent. ${ }^{17)}$ These results have shown that the dye binds to $E$. coli kinase by an electrostatic interaction rather than by a hydrophobic one.

Blue dextran was found to inhibit $E$. coli kinase competitively with respect to 3-phosphoglycerate, but noncompetitively with respect to ATP. Since yeast kinase has been shown to be inhibited competitively with respect to ATP, ${ }^{1)}$ there is a difference between the interaction of $E$. coli kinase and yeast kinase with blue dextran. Cibacron Blue inhibited $E$. coli kinase competitively with respect to 3-phosphoglycerate under either a saturated or an unsaturated level of ATP as a fixed substrate, but the inhibition type with respect to ATP shifted from mixed-type inhibition under a saturated level of 3phosphoglycerate to competitive inhibition under an unsaturated level. Since one molecule of Cibacron Blue binds per molecule of E. coli kinase under a saturated level of ATP, and since two molecules of the dye bind to one enzyme molecule under an unsaturated level, this shift seems to be caused by the binding of second dye molecule to $E$. coli kinase. It appears that the second dye molecule may affect the binding of ATP to the kinase. It is suggested that the ATP binding site on E. coli kinase can accommodate Cibacron Blue, but not the chromophore of blue dextran; presumably this may be due to steric hindrance by the bulky dextran matrix which surrounded Cibacron Blue. On the other hand, the ATP binding site on yeast kinase may be sufficiently open to accommodate the blue chromophore of the dextran. In addition, it is suggested that E. coli kinase is also adsorbed to blue dextranSepharose at or near the 3-phosphoglycerate 
binding site rather than at the ATP binding site, as is the case with phosphoglycerate kinase from L. plantarum. ${ }^{7}$ )

\section{REFERENCES}

1) S. T. Thompson, K. H. Cass and E. Stellwagen, Proc. Natl. Acad. Sci. U.S.A., 72, 669 (1975).

2) S. T. Thompson and E. Stellwagen, Proc. Natl. Acad. Sci. U.S.A., 73, 361 (1976).

3) E. Stellwagen, Acc. Chem. Res., 10, 92 (1977).

4) R. S. Beissner and F. B. Rudolph, Arch. Biochem. Biophys., 189, 76 (1978).

5) H. J. Böhme, G. Kopperschläger, J. Schulz and E. Hofmann, J. Chromatogr., 69, 209 (1972).

6) R. S. Beissner and F. B. Rudolph, J. Biol. Chem., 254, 6237 (1979).

7) K. Kawai and Y. Eguchi, J. Biochem., 88, 1227 (1980).

8) O. H. Lowry, N. J. Rosebrough, A. L. Farr and R. J. Randall, J. Biol. Chem., 193, 265 (1951).
9) L. D. Ryan and C. S. Vestling, Arch. Biochem. Biophys., 160, 279 (1974).

10) W. W. Cleland, Biochim. Biophys. Acta, 67, 173 (1963)

11) W. W. Cleland, "The Enzymes," Vol. II, ed. by P. D. Boyer, Academic Press, Inc., New York and London, 1970, p. 1.

12) J. Monod, J-P. Changeux and F. Jacob, J. Mol. Biol., 6, 306 (1963).

13) J-P. Changeux, Cold Spring Harbor Symp. Quant. Biol., 28, 497 (1963).

14) K. Kawai and Y. Eguchi, J. Ferment. Technol., 58, 383 (1980).

15) E. Stellwagen, R. Cass, S. T. Thompson and M. Woody, Nature, 257, 716 (1975).

16) G. D'Alessio and J. Josse, "Methods in Enzymology," Vol. XLII, ed. by W. A. Wood, Academic Press, Inc., New York and London, 1975, p. 139.

17) B. B. Chambers and R. B. Dunlap, J. Biol. Chem., 254, 6515 (1979). 